\title{
On hyperboundedness and spectrum of Markov operators
}

\author{
Laurent Miclo
}

\author{
Institut de Mathématiques de Toulouse, UMR 5219 \\ Université de Toulouse and CNRS, France
}

\begin{abstract}
Consider an ergodic Markov operator $M$ reversible with respect to a probability measure $\mu$ on a general measurable space. It is shown that if $M$ is bounded from $\mathbb{L}^{2}(\mu)$ to $\mathbb{L}^{p}(\mu)$, where $p>2$, then it admits a spectral gap. This result answers positively a conjecture raised by Høegh-Krohn and Simon [31] in a semi-group context. The proof is based on isoperimetric considerations and especially on Cheeger inequalities of higher order for weighted finite graphs recently obtained by Lee, Gharan and Trevisan [23]. It provides a quantitative link between hyperboundedness and an eigenvalue different from the spectral gap in general. In addition, the usual Cheeger inequality is extended to the higher eigenvalues in the compact Riemannian setting.
\end{abstract}

Keywords: Markov operators and semi-groups, hyperboundedness, spectral gap, Cheeger's inequalities, (Dirichlet) connectivity spectrum, Orlicz's norms, hypercontractivity.

MSC2010: first: 60J25, secondary: 47A30, 46E30, 47A75, 37A30, 58J50, 05C50. 


\section{Introduction}

The main purpose of this article is to show that hyperbounded, ergodic and self-adjoint Markov operators admit a spectral gap. This property solves a conjecture raised by Høegh-Krohn and Simon [31] in a semi-group context.

More precisely, let us start with a probability space $(S, \mathcal{S}, \mu)$. A self-adjoint operator $M$ : $\mathbb{L}^{2}(\mu) \rightarrow \mathbb{L}^{2}(\mu)$ is said to be Markovian if

$$
\begin{aligned}
\forall f \in \mathbb{L}^{2}(\mu), \quad f \geqslant 0 & \Rightarrow M[f] \geqslant 0 \\
M[\mathbb{1}] & =\mathbb{1}
\end{aligned}
$$

where $\mathbb{1}$ is the function always taking the value 1 and where all the previous statements have to be understood $\mu$-almost surely.

In particular, $M$ admits a spectral decomposition: there exists a projection-valued measure $\left(E_{l}\right)_{l \in[-1,1]}$ such that

$$
M=\int_{-1}^{1} l d E_{l}
$$

(see e.g. the chapter 7 of the book of Reed and Simon [30]).

The Markov operator $M$ is said to be ergodic if

$$
\forall f \in \mathbb{L}^{2}(\mu), \quad M f=f \Rightarrow f \in \operatorname{Vect}(\mathbb{1})
$$

namely if $\left(E_{1}-E_{1-}\right)\left[\mathbb{L}^{2}(\mu)\right]$ is reduced to $\operatorname{Vect}(\mathbb{1})$, the vector line generated by $\mathbb{1}$.

This property is implied by the following stronger requirement: $M$ has a spectral gap if there exists $\lambda>0$ such that $\left(E_{1}-E_{1-\lambda}\right)\left[\mathbb{L}^{2}(\mu)\right]=\operatorname{Vect}(\mathbb{1})$ and by definition the associated spectral gap is the supremum of such $\lambda$.

Finally, the Markov operator $M$ is said to be hyperbounded if there exists $p>2$ such that

$$
\|M\|_{\mathbb{L}^{2}(\mu) \rightarrow \mathbb{L}^{p}(\mu)}<+\infty
$$

We can now state the objective of this paper:

Theorem 1 If a self-adjoint Markovian operator is ergodic and hyperbounded then it admits a spectral gap.

This result was conjectured by Høegh-Krohn and Simon [31], who rather believed it to be wrong, in the more restricted context of semi-groups. They started with a continuous family of self-adjoint Markovian operators $P:=\left(P_{t}\right)_{t \geqslant 0}$ defined on $\mathbb{L}^{2}(\mu)$ and satisfying the semi-group property: $P_{0}$ is the identity operator and

$$
\forall t, s \geqslant 0, \quad P_{t} P_{s}=P_{t+s}
$$

The semi-group $P$ is said to be ergodic if for any $f \in \mathbb{L}^{2}(\mu), P_{t}[f]$ converges in $\mathbb{L}^{2}(\mu)$ toward $\mu[f]$ (seen as the function $\mu[f] \mathbb{1}$ ). It is said to have a spectral gap if the previous convergence is uniform over the unit ball of $\mathbb{L}^{2}(\mu)$. These properties can be characterized through the associated generator $L$ (see for instance the book of Yosida [37]): it is the self-adjoint operator defined on the dense domain $\mathcal{D}(L)$ of functions $f \in \mathbb{L}^{2}(\mu)$ such that $\left(P_{t}[f]-f\right) / t$ converges in $\mathbb{L}^{2}(\mu)$ as $t$ goes to $0_{+}$. By definition, the limit is $L[f]$. The self-adjoint operator $L$ is non-positive definite and can be spectrally decomposed: there exists a projection-valued measure $\left(F_{l}\right)_{l \in \mathbb{R}_{+}}$such that

$$
L=-\int_{\mathbb{R}_{+}} l d F_{l}
$$


By functional calculus, we then have

$$
\forall t \geqslant 0, \quad P_{t}=\int_{\mathbb{R}_{+}} \exp (-t l) d F_{l}
$$

Ergodicity and the existence of a spectral gap respectively amount to $F_{0}\left[\mathbb{L}^{2}(\mu)\right]=$ Vect $(\mathbb{1})$ and to the existence of $\lambda>0$ such that $F_{\lambda}\left[\mathbb{L}^{2}(\mu)\right]=\operatorname{Vect}(\mathbb{1})$. By definition, the spectral gap is the supremum of such $\lambda$. It can also be computed via the corresponding Dirichlet form: for any $f \in \mathbb{L}^{2}(\mu)$, the mapping $\mathbb{R}_{+}^{*} \ni t \mapsto \mu\left[f\left(\mathrm{Id}-P_{t}\right)[f]\right] / t$ is nonincreasing, designate by $\mathcal{E}(f)$ its limit in $0_{+}$, which belongs to $\overline{\mathbb{R}}_{+}$. This quantity is called the energy of $f$ and let $\mathcal{D}(\mathcal{E}):=\left\{f \in \mathbb{L}^{2}(\mu)\right.$ : $\mathcal{E}(f)<\infty\}$ (it appears that $\mathcal{D}(L) \subset \mathcal{D}(\mathcal{E})$ and for $f \in \mathcal{D}(L), \mathcal{E}(f)=-\mu[f L[f]]$ ). The mapping $\mathcal{D}(\mathcal{E}) \ni f \mapsto \mathcal{E}(f)$ is referred to as the Dirichlet form associated to $P$ (see for instance the book [12] of Fukushima, Öshima and Takeda). The semi-group $P$ admits a spectral gap if and only if the following quantity $\lambda_{2}$ is nonzero, in which case it is the spectral gap of $P$ :

$$
\lambda_{2}:=\left(\sup _{f \in \mathbb{L}^{2}(\mu)} \frac{\operatorname{Var}(f, \mu)}{\mathcal{E}(f)}\right)^{-1}
$$

where $\operatorname{Var}(f, \mu):=\mu\left[f^{2}\right]-(\mu[f])^{2}$ is the variance of $f$ with respect to $\mu$. In the above expression, the convention $0 \cdot \infty:=0$ is enforced as usual. Finally, the semi-group $P$ is said to be hyperbounded, if there exists a time $T \geqslant 0$ such that the Markov operator $P_{T}$ is hyperbounded in the previous sense.

Høegh-Krohn and Simon [31] were wondering if hyperbounded, ergodic and continuous selfadjoint Markov semi-groups admit a spectral gap. Theorem 1 enables to answer positively to this question. It is sufficient to apply it to an element $P_{T}$ of the semi-group which is hyperbounded. The Markov operator $P_{T}$ is seen to be ergodic via the representation (1).

Let us mention that the Høegh-Krohn and Simon's conjecture is easy to solve, if the hyperboundedness assumption is strengthened into hypercontractivity: the continuous self-adjoint Markov semi-group is said to be hypercontractive, if there exist $p>2$ and a time $T \geqslant 0$ such that the norm of $P_{T}$ from $\mathbb{L}^{2}(\mu)$ to $\mathbb{L}^{p}(\mu)$ is 1 . It is well-known that such a semi-group admits a spectral gap, and better, its generator satisfies a logarithmic Sobolev inequality, property in fact equivalent to hypercontractivity. Hyperboundedness is itself equivalent to a non-tight logarithmic Sobolev inequality. The existence of a spectral gap enables to tight such an inequality (for all the previous assertions, see for instance the book of Ané, Blachère, Chafai,, Fougères, Gentil, Malrieu, Roberto and Scheffer [4]). Thus Theorem 1 shows that for semi-groups, hyperboundedness is equivalent of hypercontractivity, but as it will appear in Proposition 10 of Section 6, there is no quantitative way to go from the former to the latter in general.

Several attempts have been made to find a counterexample or to prove Høegh-Krohn and Simon's conjecture, but extra assumptions were always needed, see for instance the papers of Aida [1], Mathieu [25], Wu [36], Hino [19, 20], Cattiaux [8], Wang [34], Gong and Wu [13] and the bibliographical comments given in section 5.9 of the book [35] of Wang for further motivations. The latter author has also shown in [34] that Theorem 1 is true if $\|M\|_{\mathbb{L}^{2}(\mu) \rightarrow \mathbb{L}^{4}(\mu)}^{4}<2$ and that it is wrong if the Markovian (or the ergodicity) assumption is removed. It provides a glimpse of the deep connection between Markovianity and isoperimetry, on which is based our approach. It is different from the various methods proposed by the above mentioned articles. A crucial ingredient is the Cheeger inequalities of higher order recently proven by Lee, Gharan and Trevisan [23] for weighted finite graphs. They are recalled in the next section, where we are to work on estimates in the finite framework. The approximation procedure enabling to treat the general situation is presented in the third section. The three last sections are devoted to further observations, respectively about general higher order Cheeger's inequalities, the extension to Orlicz spaces and a quantitative version of Theorem 1. An appendix adapts to principal Dirichlet eigenvalues some estimates of Holley, 
Kusuoka and Stroock [21] relative to spectral gaps at small temperature. They are required by an illustration of the interest of the Dirichlet connectivity spectra defined in Section 4.

\section{Higher order Cheeger inequalities in the finite set- ting}

In this section we deduce a lower bound on the $\mathbb{L}^{2}$ to $\mathbb{L}^{p}$ operator norm of finite Markov kernels in terms of their spectrum, for $p>2$.

So here $S$ is a finite set of cardinal $N \in \mathbb{N} \backslash\{1\}$ (endowed with the trivial $\sigma$-field) and $\mu$ is a (strictly) positive probability measure on $S$. We start with a Markovian generator $L$, namely a matrix $(L(x, y))_{x, y \in S}$ whose off-diagonal entries are non-negative and whose lines sum up to zero. We assume that $\mu$ is reversible with respect to $L$, in the sense that

$$
\forall x, y \in S, \quad \mu(x) L(x, y)=\mu(y) L(y, x)
$$

It means that seen as an operator, $L$ is symmetric in $\mathbb{L}^{2}(\mu)$. It is also well-known to be non-positive definite, so let us write down the spectrum of $-L$ as

$$
0=\lambda_{1} \leqslant \lambda_{2} \leqslant \cdots \leqslant \lambda_{N}
$$

(the first equality comes from the fact that the kernel of $L$ contains at least $\operatorname{Vect}(\mathbb{1})$ ).

Cheeger's inequality in the finite setting relates $\lambda_{2}$ to an isoperimetric quantity. To recall it, we introduce the conductance associated to any subset $A \subset S$ with $A \neq \varnothing$ :

$$
j(A):=\frac{\mu\left(\mathbb{1}_{A} L\left(\mathbb{1}_{A^{\mathrm{c}}}\right)\right)}{\mu(A)}
$$

(where $\mathbb{1}_{A}$ is the indicator function of $A$ ). This quantity if of "isoperimetric" nature, since the numerator is a measurement of the frontier between $A$ and $A^{\mathrm{c}}$, its value is $\sum_{x \in A, y \notin A} \mu(x) L(x, y)$, while the denominator is the volume of $A$. The connectivity constant of $L$ is then defined by

$$
\iota_{2}:=\min _{A \neq \varnothing, S} \max \left\{j(A), j\left(A^{\mathrm{c}}\right)\right\}=\min _{A: 0<\mu(A) \leqslant 1 / 2} j(A)
$$

The Cheeger's inequality states that

$$
\frac{\iota_{2}^{2}}{8|L|} \leqslant \lambda_{2} \leqslant \iota_{2}
$$

with $|L|:=\max _{x \in S}|L(x, x)|$ (if $|L|$ happens to vanish, it means that $L=0$, so we can assume that $|L|>0$ to avoid trivial statements). It was first obtained by Cheeger [9] in a compact Riemannian manifold setting. Its extension to finite weighted graphs, or equivalently to finite reversible Markov processes, is due to Alon and Milman [3], Alon [2], Lawler and Sokal [22] and Sinclair and Jerrum $[32]$.

It is natural to wonder if a variant of this result would hold for the other eigenvalues $\lambda_{3}, \ldots$, $\lambda_{N}$. It leads to introduce the following connectivity spectrum $\left(\iota_{n}\right)_{n \in \llbracket N \rrbracket}$. For $n \in \mathbb{N}$, let $\mathcal{D}_{n}$ be the set of $n$-tuples $\left(A_{1}, \ldots, A_{n}\right)$ of disjoint and non-empty subsets of $S$. Define

$$
\forall n \in \llbracket N \rrbracket, \quad \iota_{n}:=\min _{\left(A_{1}, \ldots, A_{n}\right) \in \mathcal{D}_{n}} \max _{k \in \llbracket n \rrbracket} j\left(A_{k}\right)
$$

Clearly $\iota_{1}=0=\lambda_{1}$ and it is not difficult to check that for $n=2$, one recovers the quantity defined in (4): $\mathcal{D}_{2}$ can be replaced by its subset containing only the partitions of $S$ into two proper subsets. 
In [27] (see also [10]), we made the conjecture that there exists a mapping $c: \mathbb{N} \rightarrow \mathbb{R}_{+}^{*}$ such that for all $(S, \mu, L)$ as above,

$$
c(n) \frac{\iota_{n}^{2}}{|L|} \leqslant \lambda_{n} \leqslant \iota_{n}
$$

The second inequality is immediate, it amounts to consider the vector space generated by the indicator functions of $n$ disjoints subsets in the variational characterization of $\lambda_{n}$ through Rayleigh quotients.

The first inequality was recently shown by Lee, Gharan and Trevisan [23] (a related result was obtained by Louis, Raghavendra, Tetali and Vempala [24], it could also be used to prove Høegh-Krohn and Simon's conjecture by slightly modifying the arguments that will follow):

Theorem 2 ([23]) There exists a universal constant $\eta>0$ such that (6) is satisfied with

$$
\forall n \in \mathbb{N}, \quad c(n):=\frac{\eta}{n^{8}}
$$

\section{Proof}

It is just a rewriting of Theorem 3.8 of [23], where the authors rather work with weighted finite graphs $(V, E, \omega): V$ is the finite set of vertices, $E$ is the set of undirected edges (which may contain loops) and $\omega: E \rightarrow \mathbb{R}_{+}$is the weight. The mapping $\omega$ is extended to $V$ by

$$
\forall v \in V, \quad \omega(v):=\sum_{u \in V:\{v, u\} \in E} \omega(\{v, u\})
$$

Lee, Gharan and Trevisan are interested in the normalized Laplacian $\mathcal{L}$ which corresponds to the $(V \times V)$-symmetric matrix

$$
\mathcal{L}:=\operatorname{Id}-D^{-1 / 2} A D^{-1 / 2}
$$

where $D$ is the diagonal matrix with entries $(\omega(v))_{v \in V}$ and $A$ is the weighted adjacency matrix $(\omega(u, v))_{u, v \in V}$. Denote by $0=\widetilde{\lambda}_{1} \leqslant \widetilde{\lambda}_{2} \leqslant \cdots \leqslant \widetilde{\lambda}_{N}$ its eigenvalues, where $N$ is the cardinal of $V$. Lee, Gharan and Trevisan also consider the connectivity spectrum $\left(\widetilde{\iota}_{n}\right)_{n \in \llbracket N \rrbracket}$, which is defined similarly to (5), but with the mapping $j$ replaced by

$$
\forall A \subset V, A \neq \varnothing, \quad \tilde{\jmath}(A) \quad:=\frac{\sum_{u \in A, v \notin A:\{u, v\} \in E} \omega(\{u, v\})}{\sum_{w \in A} \omega(w)}
$$

Then Theorem 3.8 of [23] states that there exists a universal constant $\eta>0$ such that

$$
\forall n \in \llbracket N \rrbracket, \quad \frac{\eta}{n^{8}} \widetilde{\iota}_{n}^{2} \leqslant \tilde{\lambda}_{n} \leqslant 2 \widetilde{\iota}_{n}
$$

To come back to the setting of Theorem 2, consider the following allocations

$$
\begin{aligned}
V & :=S \\
E & :=\{\{x, y\}: L(x, y)>0 \text { or } x=y\} \\
\forall e \in E, \quad \omega(e) & := \begin{cases}\mu(x) L(x, y) & \text { if } e=\{x, y\} \text { with } x \neq y \\
|L|+L(x, x) & \text {, if } e=\{x, x\}\end{cases}
\end{aligned}
$$

We get that

$$
\forall x \in S, \quad \omega(x)=|L| \mu(x)
$$


and it follows that for all $n \in \llbracket N \rrbracket, \widetilde{\iota}_{n}=\iota_{n} /|L|$. Furthermore, by the variational characterization of the eigenvalues (see Equation (9) of [23]), we have

$$
\begin{aligned}
\tilde{\lambda}_{n} & =\frac{1}{2} \min _{H: \operatorname{dim}(H)=n} \max \left\{\frac{\sum_{x, y \in S} \omega(\{x, y\})(f(y)-f(x))^{2}}{\sum_{z \in S} \omega(z) f^{2}(z)}: f \in H \backslash\{0\}\right\} \\
& =\frac{\lambda_{n}}{|L|}
\end{aligned}
$$

(where the minimum is taken over all subspaces $H$ of dimension $n$ of $\mathbb{L}^{2}(\mu)$ ). The announced result is now an immediate consequence of (7).

Lee, Gharan and Trevisan [23] have proposed several improvements of (7), see Remark 8 and Theorem 12 below. Nevertheless, as it will be clear in the next section, the exact expression of $c(n)$ for $n \in \mathbb{N}$ is not important for the purpose of proving Theorem 1, what really matters is that $c(n)>0$. But we will come back to this question in Section 6 below.

Even if at first view hyperboundedness does not seem a very pertinent notion in the finite setting, let us derive from Theorem 2 a quantitative bound for this property. As in the introduction, we consider on the finite set $S$ a Markov kernel $M$ which is symmetric in $\mathbb{L}^{2}(\mu)$. Denote its spectrum by

$$
1=\theta_{1} \geqslant \theta_{2} \geqslant \cdots \geqslant \theta_{N} \geqslant-1
$$

Proposition 3 Assume that for some $n \in \llbracket N \rrbracket$, we have $\theta_{n} \geqslant 1-c(n) / 4$. Then we can deduce that for any $p>2$,

$$
\|M\|_{\mathbb{L}^{2}(\mu) \rightarrow \mathbb{L}^{p}(\mu)}^{p} \geqslant \frac{\left(1-2 \delta_{n}\right)^{p}}{2} n^{\frac{p}{2}-1}
$$

where $\delta_{n}:=\sqrt{\left(1-\theta_{n}\right) / c(n)} \leqslant 1 / 2$.

\section{Proof}

To come back to the situation of Theorem 2, we introduce the Markovian generator $L=M-\mathrm{Id}$, where Id is the $(S \times S)$-identity matrix. We have $|L| \leqslant 1$ and the spectrum of $-L$ defined in (2) is given by

$$
\forall m \in \llbracket N \rrbracket, \quad \lambda_{m}=1-\theta_{m}
$$

Next consider $n \in \llbracket N \rrbracket$ as in the statement of the above proposition. According to Theorem 2, we have

$$
\iota_{n} \leqslant \sqrt{|L| \lambda_{n} / c(n)} \leqslant \sqrt{\lambda_{n} / c(n)}=\delta_{n}
$$

so we can find $\left(A_{1}, \ldots, A_{n}\right) \in \mathcal{D}_{n}$ satisfying,

$$
\forall k \in \llbracket n \rrbracket, \quad \delta_{n} \mu\left(A_{k}\right) \geqslant \mu\left[\mathbb{1}_{A_{k}} L\left[\mathbb{1}_{A_{k}^{\mathrm{c}}}\right]\right]
$$

Taking into account that

$$
\begin{aligned}
L\left[\mathbb{1}_{A_{k}^{\mathrm{c}}}\right] & =M\left[\mathbb{1}_{A_{k}^{\mathrm{c}}}\right]-\mathbb{1}_{A_{k}^{\mathrm{c}}} \\
& =M\left[\mathbb{1}-\mathbb{1}_{A_{k}}\right]-\mathbb{1}_{A_{k}^{\mathrm{c}}} \\
& =\mathbb{1}-M\left[\mathbb{1}_{A_{k}}\right]-\mathbb{1}_{A_{k}^{\mathrm{c}}}
\end{aligned}
$$


we deduce that for any $k \in \llbracket n \rrbracket$,

$$
\left(1-\delta_{n}\right) \mu\left(A_{k}\right) \leqslant \mu\left[\mathbb{1}_{A_{k}} M\left[\mathbb{1}_{A_{k}}\right]\right]
$$

For any $k \in \llbracket n \rrbracket$, consider the set $B_{k}:=\left\{x \in A_{k}: M\left[\mathbb{1}_{A_{k}}\right](x) \geqslant 1-2 \delta_{n}\right\}$. We compute that

$$
\begin{aligned}
\mu\left[\mathbb{1}_{A_{k}} M\left[\mathbb{1}_{A_{k}}\right]\right] & =\mu\left[\mathbb{1}_{B_{k}} M\left[\mathbb{1}_{A_{k}}\right]\right]+\mu\left[\mathbb{1}_{A_{k} \backslash B_{k}} M\left[\mathbb{1}_{A_{k}}\right]\right] \\
& \leqslant \mu\left(B_{k}\right)+\left(1-2 \delta_{n}\right)\left(\mu\left(A_{k}\right)-\mu\left(B_{k}\right)\right)
\end{aligned}
$$

It follows from the two last bounds that

$$
\frac{1}{2} \mu\left(A_{k}\right) \leqslant \mu\left(B_{k}\right) \leqslant \mu\left(A_{k}\right)
$$

Since the sets $A_{1}, \ldots, A_{n}$ are disjoint, there exists $k \in \llbracket n \rrbracket$ such that $\mu\left(A_{k}\right) \leqslant 1 / n$. Consider $f=\mathbb{1}_{A_{k}}$, it appears that

$$
\mu\left[f^{2}\right]=\mu\left(A_{k}\right)
$$

and since by assumption $1-2 \delta_{n} \geqslant 0$, we get by definition of $B_{k}$,

$$
\begin{aligned}
\mu\left[|M[f]|^{p}\right] & \geqslant\left(1-2 \delta_{n}\right)^{p} \mu\left(B_{k}\right) \\
& \geqslant \frac{\left(1-2 \delta_{n}\right)^{p}}{2} \mu\left(A_{k}\right)
\end{aligned}
$$

In particular, we obtain that

$$
\begin{aligned}
\|M\|_{\mathbb{L}^{2}(\mu) \rightarrow \mathbb{L}^{p}(\mu)}^{p} & \geqslant \frac{\mu\left[|M[f]|^{p}\right]}{\mu\left[f^{2}\right]^{\frac{p}{2}}} \\
& \geqslant \frac{\left(1-2 \delta_{n}\right)^{p}}{2 \mu\left(A_{k}\right)^{\frac{p}{2}-1}} \\
& \geqslant \frac{\left(1-2 \delta_{n}\right)^{p}}{2} n^{\frac{p}{2}-1}
\end{aligned}
$$

as announced.

\section{An approximation procedure}

We come back to the Høegh-Krohn and Simon's conjecture framework and approximate it by finite sets.

The proof of Theorem 1 relies on a contradictory argument, explaining why it does not provide a quantitative estimation of the spectral gap in terms of the hyperbounded operator norm. Indeed this is not possible, as it will be seen in the last section. With the notations of the introduction, our starting observation is:

Lemma 4 The ergodic self-adjoint Markov operator $M$ has no spectral gap if and only if for any $\lambda>0,\left(E_{1}-E_{1-\lambda}\right)\left[\mathbb{L}^{2}(\mu)\right]$ is of infinite dimension.

\section{Proof}

By the requirements imposed on the projection-valued family $\left(E_{\lambda}\right)_{\lambda \in[-1,1]}$ in the spectral Theorem (see for instance the chapter 7 of the book of Reed and Simon [30]), for any $f \in \mathbb{L}^{2}(\mu)$, the mapping $[-1,1] \ni \lambda \mapsto \mu\left[f E_{\lambda}[f]\right]$ is non-decreasing. It follows that the mapping $[0,2] \ni \lambda \mapsto$ 
$\mu\left[f\left(E_{1}-E_{1-\lambda}\right)[f]\right]$ is non-decreasing. Since for any $\lambda \in[0,2], E_{1}-E_{\lambda}$ is a projection operator, we get that the mapping $[0,2] \ni \lambda \mapsto \operatorname{dim}\left(\left(E_{1}-E_{1-\lambda}\right)\left[\mathbb{L}^{2}(\mu)\right]\right)$ is non-decreasing. So if for some $\lambda \in(0,2]$, we have that $\operatorname{dim}\left(\left(E_{1}-E_{1-\lambda}\right)\left[\mathbb{L}^{2}(\mu)\right]\right)<+\infty$, it appears that the $\mathbb{Z}_{+}$-valued mapping $[0, \lambda] \ni l \mapsto \operatorname{dim}\left(E_{1}-E_{1-l}\right)$ has a finite number of jumps, say $0=l_{1}<l_{2}<\cdots l_{r} \leqslant \lambda$. Each of them corresponds to an eigenvalue $1-l_{i}, i \in \llbracket r \rrbracket$, whose multiplicity is given by the height of the jump (the first one is $1=\operatorname{dim}\left(\left(E_{1}-E_{1-}\right)\left[\mathbb{L}^{2}(\mu)\right]\right)$, due to the ergodicity assumption). Then $M$ admits $l_{2}>0$ as spectral gap.

Conversely if the ergodic self-adjoint Markov operator $M$ has a spectral gap, then $\left(E_{1}-\right.$ $\left.E_{1-\lambda}\right)\left[\mathbb{L}^{2}(\mu)\right]$ is of dimension 1 for $\lambda>0$ small enough.

From now on, we assume that the ergodic self-adjoint Markov operator $M$ has no spectral gap. For any $n \in \mathbb{N}$, let $0<\epsilon_{n}<1 \wedge(c(n) / 32)$ be given, where $c(n)$ is defined in Theorem 2 . By the above lemma, we can find $f_{1}, \ldots, f_{n} \in \mathbb{L}^{2}(\mu)$, which are normalized, mutually orthogonal and so that

$$
\mu\left[f_{i} M f_{j}\right] \quad \begin{cases}=0 & , \text { if } i \neq j \\ \geqslant\left(1-\epsilon_{n}\right) & \text {,if } i=j\end{cases}
$$

Indeed, we can first take $f_{1}=\mathbb{1}$. Next we choose $f_{2}$ among the normalized functions of the infinite dimensional space

$$
\left\{g \in\left(E_{1}-E_{1-\lambda}\right)\left[\mathbb{L}^{2}(\mu)\right]: \mu\left[g f_{1}\right]=0 \text { and } \mu\left[g M\left[f_{1}\right]\right]=0\right\}
$$

Iterating this procedure leads to functions $f_{1}, \ldots, f_{n}$ satisfying the wanted properties.

To come back to the finite case, consider a non-decreasing family $\left(\mathcal{S}_{N}\right)_{N \in \mathbb{N}}$ of finite sub- $\sigma$ algebras of $\mathcal{S}$ such that

$$
\bigvee_{N \in \mathbb{N}} \mathcal{S}_{N}=\sigma\left(f_{1}, \ldots, f_{n}\right)
$$

This is possible, because the $\sigma$-algebra generated by $f_{1}, \ldots, f_{n}$ is separable. Fixing $N \in \mathbb{N}$, we consider $\mu_{N}$ the restriction of $\mu$ to $\mathcal{S}_{N}, I_{N}$ the natural injection of $\mathbb{L}^{2}\left(\mu_{N}\right)$ into $\mathbb{L}^{2}(\mu)$ and $\mathbb{E}_{N}$ the conditional expectation (projection operator) with respect to $\mathcal{S}_{N}$. Define furthermore $M_{N}:=$ $\mathbb{E}_{N} M I_{N}$, which is a reversible Markov kernel on $\left(S_{N}, \mathcal{S}_{N}, \mu_{N}\right)$, where $S_{N}$ is the finite set of atoms of $\mathcal{S}_{N}$.

By Jensen's inequality, we have for any $p>2$ and $g \in \mathbb{L}^{2}\left(\mu_{N}\right)$,

$$
\begin{aligned}
\mu_{N}\left[\left|M_{N}[g]\right|^{p}\right] & \leqslant \mu_{N}\left[\mathbb{E}_{N}\left[\left|M I_{N}[g]\right|^{p}\right]\right] \\
& =\mu\left[\left|M I_{N}[g]\right|^{p}\right]
\end{aligned}
$$

Similarly, we have $\mu_{N}\left[g^{2}\right]=\mu\left[\left(I_{N}[g]\right)^{2}\right]$, so it follows that

$$
\begin{aligned}
\left\|M_{N}\right\|_{\mathbb{L}^{2}\left(\mu_{N}\right) \rightarrow \mathbb{L}^{p}\left(\mu_{N}\right)}^{p} & \leqslant \sup \left\{\mu\left[|M[f]|^{p}\right]: f \in I_{N}\left[\mathbb{L}^{2}\left(\mu_{N}\right)\right], \mu\left[f^{2}\right]=1\right\} \\
& \leqslant\|M\|_{\mathbb{L}^{2}(\mu) \rightarrow \mathbb{L}^{p}(\mu)}^{p}
\end{aligned}
$$

Furthermore by the martingale convergence theorem, for any $f \in \mathbb{L}^{2}\left(\sigma\left(f_{1}, \ldots, f_{n}\right), \mu\right)$, we have in $\mathbb{L}^{2}(\mu)$,

$$
\lim _{N \rightarrow \infty} \mathbb{E}_{N}[f]=f
$$

and taking into account the continuity of $M$,

$$
\lim _{N \rightarrow \infty} M\left[\mathbb{E}_{N}[f]\right]=M[f]
$$


We deduce the convergence of the matrices

$$
\begin{aligned}
\lim _{N \rightarrow \infty}\left(\mu_{N}\left[\mathbb{E}_{N}\left[f_{i}\right] \mathbb{E}_{N}\left[f_{j}\right]\right]\right)_{i, j \in \llbracket N \rrbracket} & =\operatorname{Id}_{N} \\
\lim _{N \rightarrow \infty}\left(\mu_{N}\left[\mathbb{E}_{N}\left[f_{i}\right] M_{N}\left[f_{j}\right]\right]\right)_{i, j \in \llbracket N \rrbracket} & =\left(\mu\left[f_{i} M f_{j}\right]\right)_{i, j \in \llbracket N \rrbracket}
\end{aligned}
$$

where $\operatorname{Id}_{N}$ is the $(N \times N)$-identity matrix. It follows that for $N$ sufficiently large,

$$
\operatorname{dim}\left(\operatorname{Vect}\left(\mathbb{E}_{N}\left[f_{1}\right], \mathbb{E}_{N}\left[f_{2}\right], \ldots, \mathbb{E}_{N}\left[f_{n}\right]\right)\right)=n
$$

and for all $g \in \operatorname{Vect}\left(\mathbb{E}_{N}\left[f_{1}\right], \mathbb{E}_{N}\left[f_{2}\right], \ldots, \mathbb{E}_{N}\left[f_{n}\right]\right)$,

$$
\mu_{N}\left[g M_{N}[g]\right] \geqslant\left(1-2 \epsilon_{n}\right) \mu_{N}\left[g^{2}\right]
$$

In particular $M_{N}$ has $n$ eigenvalues above $1-2 \epsilon_{n}$. We can now apply Proposition 3 with $\delta_{n}:=$ $\sqrt{2 \epsilon_{n} / c(n)} \leqslant 1 / 4$, to get

$$
\begin{aligned}
\left\|M_{N}\right\|_{\mathbb{L}^{2}\left(\mu_{N}\right) \rightarrow \mathbb{L}^{p}\left(\mu_{N}\right)}^{p} & \geqslant \frac{\left(1-2 \delta_{n}\right)^{p}}{2} n^{\frac{p}{2}-1} \\
& \geqslant 2^{-p-1} n^{\frac{p}{2}-1}
\end{aligned}
$$

It follows from (10) that

$$
\|M\|_{\mathbb{L}^{2}(\mu) \rightarrow \mathbb{L}^{p}(\mu)}^{p} \geqslant 2^{-p-1} n^{\frac{p}{2}-1}
$$

and since this is true for all $n \in \mathbb{N}, M$ cannot be hyperbounded.

\section{General higher order Cheeger's inequalities}

Instead of first obtaining a hyperboundedness estimate in the finite setting and proceeding next to the approximation of the general case, the order of these two steps can be reversed. Several consequences of this observation are brought together here.

As in the introduction, let $M$ be a self-adjoint Markov operator in $\mathbb{L}^{2}(\mu)$, where $(S, \mathcal{S}, \mu)$ is a probability space. For any $n \in \mathbb{N}$, define

$$
\lambda_{n}(M):=\inf _{H: \operatorname{dim}(H)=n} \max \left\{\frac{\mu[f(\operatorname{Id}-M)[f]]}{\mu\left[f^{2}\right]}: f \in H \backslash\{0\}\right\}
$$

where the infimum is taken over all subspaces $H$ of $\mathbb{L}^{2}(\mu)$ of dimension $n$ (by usual conventions, $\lambda_{n}=+\infty$ for $n$ is strictly larger than the dimension of $\mathbb{L}^{2}(\mu)$ ). In the general framework, these quantities are no longer necessarily counting the ordered eigenvalues of $\operatorname{Id}-M$ with their multiplicities, for instance if $M$ is ergodic and has no spectral gap, then $\lambda_{n}=0$ for all $n \in \mathbb{N}$, whereas the dimension of the eigenspace associated to 0 is 1.

Definition (3) can be extended to all non-negligible and measurable $A \in \mathcal{S}$ :

$$
j(A):=\frac{\mu\left(\mathbb{1}_{A} M\left(\mathbb{1}_{A^{\mathrm{c}}}\right)\right)}{\mu(A)}
$$

which in turn leads to introduce the connectivity spectrum $\left(\iota_{n}(M)\right)_{n \in \mathbb{N}}$ through (5) (where $\mathcal{D}_{n}$ stands now for set of $n$-tuples of disjoint and non-negligible elements of $\mathcal{S}$ ).

Let us also consider

$$
|M|:=\operatorname{esssup}_{x \in S} \max _{A \in \mathcal{S}: x \notin A} M\left[\mathbb{1}_{A}\right](x)
$$

(where the essential supremum is relative to $\mu$ ). Under mild regularity assumptions, this quantity takes the form $\sup _{x \in S} M(x, S \backslash\{x\})$, which in continuous settings is often equal to 1 and thus can be removed from the following result.

The approximation procedure described in the previous section then leads without difficulty to: 
Proposition 5 With $\eta>0$ the universal constant of Theorem 2, we have

$$
\forall n \in \mathbb{N}, \quad \frac{\eta}{n^{8}} \frac{\iota_{n}^{2}(M)}{|M|} \leqslant \lambda_{n}(M) \leqslant \iota_{n}(M)
$$

As a consequence, Proposition 3 can be proven in the general setting by the same arguments and Theorem 1 follows directly.

It is tempting to extend the above proposition to Markovian generators. So let $\left(P_{t}\right)_{t \geqslant 0}$ be a continuous self-adjoint Markovian semi-group, as after the statement of Theorem 1. Denote by $L$ its generator (in $\mathbb{L}^{2}(\mu)$ ). Since it is a non-positive self-adjoint (but in general non-bounded) operator, we can apply spectral functional calculus to see that if we define for any $n \in \mathbb{N}$,

$$
\lambda_{n}(L):=\inf _{H: \operatorname{dim}(H)=n} \max \left\{\frac{\mu[f(-L)[f]]}{\mu\left[f^{2}\right]}: f \in H \backslash\{0\}\right\}
$$

(where the infimum is taken over subspaces $H$ of the domain $\mathcal{D}(L) \subset \mathbb{L}^{2}(\mu)$ of $L$ ), then we have

$$
\begin{aligned}
\forall n \in \mathbb{N}, \quad \lambda_{n}(L) & =\lim _{t \rightarrow 0_{+}} \frac{1-\exp \left(-t \lambda_{n}(L)\right)}{t} \\
& =\lim _{t \rightarrow 0_{+}} \frac{\lambda_{n}\left(P_{t}\right)}{t}
\end{aligned}
$$

A priori, the definition of a connectivity spectrum $\left(\iota_{n}(L)\right)_{n \in \mathbb{N}}$ associated to $L$ is less obvious. First we note that for any $A \in \mathcal{S}$, the mapping

$$
(0,+\infty) \ni t \mapsto \frac{1}{t} \mu\left[\mathbb{1}_{A} P_{t}\left[\mathbb{1}_{A^{\mathrm{c}}}\right]\right]=\frac{1}{t} \mu\left[\mathbb{1}_{A}\left(\mathrm{Id}-P_{t}\right)\left[\mathbb{1}_{A}\right]\right]
$$

is non-increasing, so we could consider its limit at $0_{+}$. Unfortunately, this limit is $+\infty$ if $\mathbb{1}_{A}$ is not in the domain $\mathcal{D}(\mathcal{E})$ of the Dirichlet form $\mathcal{E}$ corresponding to $L$. This is very restrictive in the continuous framework, because for $\mathbb{1}_{A} \in \mathcal{D}(\mathcal{E}), \mathbb{1}_{A}$ must be quasi-continuous: for instance if $L$ is the Laplacian on [0,1] with Neumann boundary conditions, then only $A=\varnothing$ and $A=[0,1]$ satisfy this condition. To avoid these problems, it is convenient to introduce the Dirichlet connectivity spectrum, which in some sense is intermediary between the usual spectrum and the connectivity spectrum. It was considered in the finite setting in $[27,10]$ and in the continuous setting for LaplaceBeltrami operators on Euclidian or Riemannian subdomains with Dirichlet boundary conditions, for instance by Helffer, Hoffmann-Ostenhof and Terracini [15] (see also the references therein).

So let us come back to a general self-adjoint Markov operator $M$ as in the beginning of this section. To any non-negligible $A \in \mathcal{S}$, we associate its first Dirichlet eigenvalue $\lambda_{0}(M, A)$ given by

$$
\left\{\begin{aligned}
\lambda_{0}(M, A) & :=\inf _{f \in \mathcal{D}(A)} \frac{\mu[f(\operatorname{Id}-M)[f]]}{\mu\left[f^{2}\right]} \\
\mathcal{D}(A) & :=\left\{f \in \mathbb{L}^{2}(\mu): f=0 \mu \text {-a.s. on } A^{\mathrm{c}}\right\}
\end{aligned}\right.
$$

Replacing $j(A)$ by $\lambda_{0}(M, A)$ in (5), we define the Dirichlet connectivity spectrum $\left(\Lambda_{n}(M)\right)_{n \in \mathbb{N}}$ of $M$ via

$$
\forall n \in \mathbb{N}, \quad \Lambda_{n}(M):=\min _{\left(A_{1}, \ldots, A_{n}\right) \in \mathcal{D}_{n}} \max _{k \in \llbracket n \rrbracket} \lambda_{0}\left(M, A_{k}\right)
$$

(again $\mathcal{D}_{n}$ stands now for set of $n$-tuples of disjoint and non-negligible elements of $\mathcal{S}$ ).

In the finite setting, as in Section 2, we can deduce from Theorem 3.7 of Lee, Gharan and Trevisan [23]:

Theorem 6 ([23]) There exists a universal constant $\hat{\eta}>0$ such that for any finite self-adjoint Markov operator $M$, we have

$$
\forall n \in \mathbb{N}, \quad \frac{\widehat{\eta}}{n^{6}} \Lambda_{n}(M) \leqslant \lambda_{n}(M) \leqslant \Lambda_{n}(M)
$$


The approximation procedure described in the previous section can easily be applied to the Dirichlet connectivity spectrum, so that the finiteness assumption can be removed from the above theorem.

We now come back to the situation of a continuous self-adjoint Markovian semi-group $\left(P_{t}\right)_{t \geqslant 0}$. For any non-negligible $A \in \mathcal{S}$ and $t \geqslant 0$, define the operator

$$
P_{A, t}: \mathbb{L}^{2}(\mu) \ni f \mapsto \mathbb{1}_{A} P_{t}\left[\mathbb{1}_{A} f\right]
$$

and consider $\mu_{A}$ the conditional expectation of $\mu$ on $A$ (for any $B \in \mathcal{S}, \mu_{A}(B)=\mu(B \cap A) / \mu(A)$ ). It appears that $\left(P_{A, t}\right)_{t \geqslant 0}$ is a continuous self-adjoint semi-group in $\mathbb{L}^{2}\left(\mu_{A}\right)$, which is subMarkovian in the sense that for all $t \geqslant 0, P_{A, t}[\mathbb{1}] \leqslant \mathbb{1}$. It admits a generator $L_{A}$, self-adjoint in $\mathbb{L}^{2}\left(\mu_{A}\right)$ and densely defined on a domain $\mathcal{D}\left(L_{A}\right)$. It follows that

$$
\begin{aligned}
\lim _{t \rightarrow 0_{+}} \frac{\lambda_{0}\left(P_{t}, A\right)}{t} & =\lim _{t \rightarrow 0_{+}} \frac{1-\exp \left(-t \lambda_{0}\left(L_{A}\right)\right)}{t} \\
& =\lambda_{0}\left(L_{A}\right)
\end{aligned}
$$

where

$$
\lambda_{0}\left(L_{A}\right)=\inf _{f \in \mathcal{D}\left(L_{A}\right) \backslash\{0\}} \frac{\mu_{A}\left[f\left(-L_{A}\right)[f]\right]}{\mu_{A}\left[f^{2}\right]}
$$

Note that the convergence in (13) is non-decreasing (as $t$ is decreasing to $0_{+}$), so that it is not difficult to deduce that for any $n \in \mathbb{N}$,

$$
\begin{aligned}
\lim _{t \rightarrow 0+} \frac{\Lambda_{n}\left(P_{t}\right)}{t} & =\Lambda_{n}(L) \\
& :=\min _{\left(A_{1}, \ldots, A_{n}\right) \in \mathcal{D}_{n}} \max _{k \in \llbracket n \rrbracket} \lambda_{0}\left(L_{A_{k}}\right)
\end{aligned}
$$

Let us point out that the quantities $\lambda_{0}\left(L_{A}\right)$ can be related more directly to $L$ and $A$ : $\mathcal{D}\left(L_{A}\right)$ is just the subspace of functions $f$ from $\mathcal{D}(L)$ which vanish on $A^{\mathrm{c}}$ and for such functions, $L_{A}[f]=\mathbb{1}_{A} L[f]$. So similarly to (12), we have $\lambda_{0}\left(L_{A}\right)=\lambda_{0}(L, A)$ with

$$
\left\{\begin{aligned}
\lambda_{0}(L, A) & :=\inf _{f \in \mathcal{D}(L, A)} \frac{\mu[f(-L)[f]]}{\mu\left[f^{2}\right]} \\
\mathcal{D}(L, A) & :=\left\{f \in \mathcal{D}(L): f=0 \mu \text {-a.s. on } A^{\mathrm{c}}\right\}
\end{aligned}\right.
$$

With these definitions, Theorem 6 extends to the generator $L$. From there it is possible to go in the direction of corresponding higher-order Cheeger inequalities. But for this paper, let us escape from the technicalities of the general Dirichlet forms and return to the original Riemannian setting of Cheeger [9]. The state space $S$ is now a compact Riemannian manifold and $L$ is the associated Laplacian $\triangle$ operator, so up to a scaling $1 / 2$ in time, $\left(P_{t}\right)_{t \geqslant 0}$ is the heat semi-group. Due to its regularizing properties, for any $t>0$, to compute $\Lambda_{n}\left(P_{t}\right)$ we can replace $\mathcal{D}_{n}$ by $\widehat{\mathcal{D}}_{n}$ the set of $n$ tuples of disjoint and open subsets of $S$ whose boundaries are smooth. For $n \geqslant 2$, we furthermore impose on $\widehat{\mathcal{D}}_{n}$ the following restrictions, which does not modify the computation of $\Lambda_{n}(\triangle)$ and will be convenient later on: each $n$-tuple $\left(A_{1}, \ldots, A_{n}\right) \in \widehat{\mathcal{D}}_{n}$ is such that for all $k \in \llbracket n \rrbracket, A_{k}$ is connected (otherwise replace $A_{k}$ by its connected component $B_{k}$ satisfying $\lambda_{0}\left(\triangle, A_{k}\right)=\lambda_{0}\left(\triangle, B_{k}\right)$ ) and each connected component of $A_{k}^{\mathrm{c}}$ contains at least one of the subsets $A_{l}$, with $l \in \llbracket n \rrbracket \backslash\{k\}$ (otherwise replace $A_{k}$ by its union with the connected components of $A_{k}^{\mathrm{c}}$ not intersecting $\sqcup_{l \in \llbracket n \rrbracket \backslash\{k\}} A_{l}$ and note that the obtained subset is still smooth and connected and its first Dirichlet eigenvalue has not increased). For $n=1$, we just assume that $A_{1} \in \widehat{\mathcal{D}}_{1}$ is connected and that its complementary set has only a finite number of connected components.

It follows from the approximation procedure (14) that the eigenvalues $\left(\lambda_{n}(\triangle)\right)_{n \in \mathbb{N}}$ of $-\triangle$ satisfy

$$
\forall n \in \mathbb{N}, \quad \frac{\widehat{\eta}}{n^{6}} \Lambda_{n}(\triangle) \leqslant \lambda_{n}(\triangle) \leqslant \Lambda_{n}(\triangle)
$$


where

$$
\Lambda_{n}(\triangle):=\min _{\left(A_{1}, \ldots, A_{n}\right) \in \hat{\mathcal{D}}_{n}} \max _{k \in \llbracket n \rrbracket} \lambda_{0}\left(\triangle, A_{k}\right)
$$

The advantage of considering regular domains is that for any $A \in \widehat{\mathcal{D}}_{1}$, it is well-known that there exists a function non-negative $f \neq 0$ in the usual Dirichlet-Sobolev space $H_{0}^{1}(A)$ such that

$$
\lambda_{0}(\triangle, A)=\frac{\int_{A}|\nabla f|^{2} d \mu}{\int_{A} f^{2} d \mu}
$$

where $\mu$ is the Riemannian probability. Up to a regularization of $(f-\epsilon)_{+}$for $\epsilon>0$ sufficiently small, we can then find a smooth non-negative function $g \neq 0$ whose support is included in $A$ such that

$$
\frac{\int_{A}|\nabla g|^{2} d \mu}{\int_{A} g^{2} d \mu} \leqslant 2 \lambda_{0}(\triangle, A)
$$

Next the traditional proof of the Cheeger inequality via the co-area formula and Sard's theorem enable to find a subset of the form $B=\{g>a\}$ with $a \in\left(0, \max _{A} g\right)$ such that

$$
\left(\frac{\sigma(\partial B)}{\mu(B)}\right)^{2} \leqslant 4 \lambda_{0}(\triangle, A)
$$

where $\partial B$ is the boundary of $B$ and $\sigma$ is the $(\operatorname{dim}(S)-1)$-dimensional measure associated to $\mu$. This observation leads to define the connectivity spectrum $\left(\iota_{n}(\triangle)\right)_{n \in \mathbb{N}}$ of $\triangle$ through

$$
\forall n \in \llbracket N \rrbracket, \quad \iota_{n}(\triangle):=\min _{\left(A_{1}, \ldots, A_{n}\right) \in \hat{\mathcal{D}}_{n}} \max _{k \in \llbracket n \rrbracket} \frac{\sigma\left(\partial A_{k}\right)}{\mu\left(A_{k}\right)}
$$

since we deduce from the above discussion the following Riemannian higher order Cheeger inequalities:

Theorem 7 There exists a universal constant $\hat{\eta}>0$ such that for any compact Riemannian manifold $S$, we have

$$
\forall n \in \mathbb{N}, \quad \lambda_{n}(\triangle) \geqslant \frac{\widehat{\eta}}{n^{6}} \iota_{n}^{2}(\triangle)
$$

By the same arguments, this result is equally valid for generators of the form $L \cdot=\triangle \cdot-\langle\nabla U, \nabla \cdot\rangle$, where $U$ is a regular potential defined on $S(\langle\cdot, \cdot\rangle$ and $\nabla$ stand for the scalar product and the gradient operator corresponding to its Riemannian structure), which are called Witten Laplacians (see for instance the book of Helffer [16]). The associated reversible probability $\mu$ admits the density proportional to $\exp (-U)$ with respect to the Riemannian measure. But let us mention why the higher order Cheeger inequalities described in Theorem 7 could turn out to be a less interesting tool than the preceding inequalities

$$
\forall n \in \mathbb{N}, \quad \frac{\widehat{\eta}}{n^{6}} \Lambda_{n}(L) \leqslant \lambda_{n}(L) \leqslant \Lambda_{n}(L)
$$

at least at "small temperature". Still in the setting of Witten Laplacian, introduce the parameter $\beta \geqslant 0$, seen as an inverse temperature, and consider the operator $L_{\beta}=\Delta \cdot-\beta\langle\nabla U, \nabla \cdot\rangle$ and the associated reversible probability $\mu_{\beta}$ whose density is proportional to $\exp (-\beta U)$. Our goal is to recover that the following convergences take place and to describe geometrically the corresponding limits $\left(l_{n}\right)_{n \in \mathbb{N}}$

$$
\forall n \in \mathbb{N}, \quad l_{n}:=-\lim _{\beta \rightarrow+\infty} \beta^{-1} \ln \left(\lambda_{n}\left(L_{\beta}\right)\right)
$$


When $U$ has a finite number of connected components of critical points, this result is due to Freidlin and Wentzell (cf. Chapter 6 of their book [11]), see also [26] for the general case, obtained by extending the approach due to Holley, Kusuoka and Stroock [21], which derived (16) for the spectral gap, namely for $n=1$. More precise descriptions of the behavior of the small eigenvalues $\lambda_{n}\left(L_{\beta}\right)$ (i.e. those for which $l_{n}>0$ ), such as the expansion of the pre-exponential factors, were proven for instance in Helffer and Nier [18] (see also Helffer, Klein and Nier [17] or Bovier, Gayrard and Klein [7] and the references given therein), but they require more work and stronger hypotheses (assuming for instance that $U$ is a Morse function).

Due to (15) (and Theorem 7 is not enough in this respect, because of the square in the r.h.s.), it is now more straightforward to deduce (16): it is sufficient to understand the behavior of $\lambda_{0}\left(L_{\beta}, A\right)$, for large $\beta \geqslant 0$, at least if this can be done in a relatively uniform manner over $A \in \widehat{\mathcal{D}}_{1}$. To proceed in this direction, we need some notations. First we remark that up to a scaling in $\beta>0$, we can and will assume from now on that $\|\nabla U\|_{\infty} \leqslant 1$ (the following arguments show that only a $\mathcal{C}^{1}$ regularity of $U$ is needed for (16)). For $x, y \in \bar{A}:=A \sqcup \partial A$, let $\mathcal{C}_{x, y}^{\bar{A}}$ be the set of continuous paths $c:[0,1] \rightarrow \bar{A}$ going from $c(0)=x$ to $c(1)=y$. The elevation of such a path $c \in \mathcal{C}_{x, y}^{\bar{A}}$ is $e(c):=\max _{t \in[0,1]} U(c(t))$ and the communication height from $x$ to $y$ in $\bar{A}$ is defined by $H(x, y):=\min _{c \in \mathcal{C}_{x, y}^{\bar{A}}} e(c)$. Finally the height of $A$ is $h(A):=\max _{x \in A, y \in \partial A} H(x, y)$. It is the height of the more profound well inside $\bar{A}$ (with respect to $U$ ): an open and connected set $B \subset S$ is said to be a well, if $U$ is constant on $\partial B$ and if for any $x \in B, U(x)<U(\partial B)$. The height of a well $B$ is given by $h(B)=U(\partial B)-\min _{B} U$. Note that $A \in \widehat{\mathcal{D}}_{1}$ does not contain a well if and only if $h(A)=0$. It will be shown in the appendix how the arguments of Holley, Kusuoka and Stroock [21] can be modified to get that there exists a constant $k_{S}>0$, depending only on the Riemannian structure of $S$, such that for all $\beta \geqslant 1$ and $A \in \widehat{\mathcal{D}}_{1}(1 / \beta)$,

$$
k_{S} \beta^{2-4 \operatorname{dim}(S)} \exp (-\beta h(A)) \leqslant \lambda_{0}\left(L_{\beta}, A\right)
$$

where for $\beta \geqslant 1, \widehat{\mathcal{D}}_{1}(1 / \beta)$ is the collection of subsets $A \in \widehat{\mathcal{D}}_{1}$ such that in each connected component of $A^{\mathrm{c}}$, we can find a point $x$ satisfying

$$
\mu\left(B(x, 1 / \beta) \cap A^{\mathrm{c}}\right) \geqslant \frac{\mu(B(x, 1 / \beta))}{2}
$$

$(B(x, 1 / \beta)$ stands for the Riemannian ball centered at $x$ and of radius $1 / \beta$ and recall that $\mu$ is the Riemannian probability of $S$ ). It is quite easy (see the appendix) to find an upper bound matching the exponential rate of (17): if for any $A \in \widehat{\mathcal{D}}_{1}$, we define

$$
\breve{k}(A):=\max \left\{\lambda_{0}\left(L_{0}, B\right): B \subset A \text { is a cycle with } h(B)=h(A)\right\}
$$

then we have

$$
\forall \beta \geqslant 1, \quad \lambda_{0}\left(L_{\beta}, A\right) \leqslant \breve{k}(A) \exp (-\beta h(A))
$$

This bound is empty if $h(A)=0$, since then by convention $\breve{k}(A)=+\infty$. In this situation, rather consider $r(A)$ the largest radius of a ball included in $A$. Then there exists a constant $k_{S}^{\prime}>0$, depending only on the Riemannian structure of $S$, such that

$$
\forall \beta \geqslant 1, \quad \lambda_{0}\left(L_{\beta}, A\right) \leqslant k_{S}^{\prime}\left(r^{-2}(A) \vee \beta^{2}\right)
$$

It will be furthermore checked in the appendix that there exists another constant $k_{S}^{\prime \prime}>0$, again depending only on the Riemannian structure of $S$, such that for any $n \geqslant 2, \beta \geqslant 1$ and $\left(A_{1}, \ldots, A_{n}\right) \in$ $\widehat{\mathcal{D}}_{n}$,

$$
\exists k \in \llbracket n \rrbracket: A_{k} \notin \widehat{\mathcal{D}}_{1}(1 / \beta) \Longrightarrow \max _{k \in \llbracket n \rrbracket} \lambda_{0}\left(L_{\beta}, A_{k}\right) \geqslant k_{S}^{\prime \prime} \beta^{2}
$$


Define

$$
\forall n \in \mathbb{N}, \quad \hat{l}_{n}:=\max _{\left(A_{1}, \ldots, A_{n}\right) \in \hat{\mathcal{D}}_{n}} \min _{k \in \llbracket n \rrbracket} h\left(A_{k}\right)
$$

It follows from the bounds (18) and (20) that if $n \geqslant 2$ is such that $\widehat{l}_{n}>0$, then for $\beta \geqslant 1$ large enough,

$$
\Lambda_{n}\left(L_{\beta}\right)=\min _{\left(A_{1}, \ldots, A_{n}\right) \in \widehat{\mathcal{D}}_{n}(1 / \beta)} \max _{k \in \llbracket n \rrbracket} \lambda_{0}\left(L_{\beta}, A_{k}\right)
$$

where $\widehat{\mathcal{D}}_{n}(1 / \beta)$ is the subset of $n$-tuples $\left(A_{1}, \ldots, A_{n}\right)$ of $\widehat{\mathcal{D}}_{n}$ consisting of elements all belonging to $\widehat{\mathcal{D}}_{1}(1 / \beta)$. It is then easy to deduce from (17) and (18) (which enables the choice of appropriate $n$-tupes from $\left.\widehat{\mathcal{D}}_{n}\right)$ that $(16)$ holds and that

$$
\forall n \in \mathbb{N} \backslash\{2\}, \quad l_{n}=\widehat{l}_{n}
$$

(this relation is also true for $n=1$, in which case both sides are trivially equal to $+\infty$ ). When $n \geqslant 2$ is such that $\widehat{l}_{n}=0$, we obtain that for any $\beta \geqslant 1$ and $A \in \widehat{\mathcal{D}}_{1}$ with $h(A)=0$,

$$
\begin{aligned}
\max \left(k_{S} \beta^{2-4 \operatorname{dim}(S)}, k_{S}^{\prime \prime} \beta^{2}\right) & \leqslant \lambda_{0}\left(L_{\beta}, A\right) \\
& \leqslant k_{S}^{\prime}\left(r^{-2}(A) \vee \beta^{2}\right)
\end{aligned}
$$

so that $(21)$ is easily seen to be equally true.

Thus generally, $l_{n}$ appears as the highest $l \geqslant 0$ such that $n$ disjoint wells of height $l$ can be found in $S$. This geometric description is also valid in the finite setting (see for instance [26]) and it was the underlying motivation for the introduction of the Dirichlet connectivity spectrum in [27], as an ersatz of "spatial localization in wells" for general Markov generators (i.e. without a small temperature parameter).

Remark 8 In their Theorem 3.7, Lee, Gharan and Trevisan [23] obtained several improvements of the factor $\hat{\eta} / n^{6}$ in Theorem 6 . First by allowing the indices $n$ of $\Lambda_{n}(M)$ and $\lambda_{n}(M)$ to be slightly different, they proved that there exists a universal constant $\eta^{\prime}>0$ such that for any $\delta \in(0,1)$ and for any finite self-adjoint Markov operator $M$,

$$
\forall n \in \mathbb{N}, \quad \frac{\eta^{\prime} \delta^{4}}{n^{2}} \Lambda_{\lceil(1-\delta) n\rceil}(M) \leqslant \lambda_{n}(M)
$$

(recall that $\lceil x\rceil$ is the smallest integer larger or equal to $x \in \mathbb{R}$ ). As usual, by the above approximation procedure, the finiteness assumption can be removed from this bound.

Next, Lee, Gharan and Trevisan [23] imposed some restrictions on the undirected graph $G$ associated to $M$ (the edges of $G$ are the $\{i, j\}$ with $i, j \in S$ such that $M(i, j)>0$ ): there exist universal constants $\eta^{\prime \prime}>0$ and $\eta^{\prime \prime \prime}>0$ such that for any $h \in \mathbb{N}, \delta \in(0,1)$ and finite self-adjoint Markov operator $M$,

- if $G$ excludes the complete graph on $h$ elements as a minor, then

$$
\forall n \in \mathbb{N}, \quad \frac{\eta^{\prime \prime} \delta^{4}}{h^{4}} \Lambda_{[(1-\delta) n]}(M) \leqslant \lambda_{n}(M)
$$

- if $G$ has genus at most $h$, then

$$
\forall n \in \mathbb{N}, \quad \frac{\eta^{\prime \prime \prime} \delta^{4}}{\ln ^{2}(1+h)} \Lambda_{\lceil(1-\delta) n\rceil}(M) \leqslant \lambda_{n}(M)
$$

Note that the previous approximation procedure is no longer sufficient to find an equivalent of these properties for the (Witten) Laplacian on a compact Riemannian manifold (a first guess would be 
that the latter estimate extends on surfaces with the corresponding notion of genus, at least if it is positive). This subject would deserve to be investigated further.

In the last section below, we will check that another improvement of Lee, Gharan and Trevisan $[23]$ is of optimal order.

\section{Other norms}

The $\mathbb{L}^{p}$ norms, $p>2$, entering Theorem 1 can be replaced by more general norms, provided they are stronger than the reference $\mathbb{L}^{2}$ norm.

More precisely, let be given on $\mathcal{F}$, the space of measurable functions on $(S, \mathcal{S})$ up to $\mu$-negligible sets, a mapping $\mathcal{N}: \mathcal{F} \rightarrow \mathbb{R}_{+} \sqcup\{+\infty\}$ which is a norm once restricted to

$$
\mathbb{L}^{\mathcal{N}}:=\{f \in \mathcal{F}: \mathcal{N}(f)<+\infty\}
$$

We assume that $\mathcal{N}$ is non-decreasing with respect to the natural order structure of $\mathbb{L}_{+}^{\mathcal{N}}:=\{f \in$ $\left.\mathbb{L}^{\mathcal{N}}: f \geqslant 0\right\}$, namely,

$$
\forall f_{1}, f_{2} \in \mathbb{L}_{+}^{\mathcal{N}}, \quad f_{1} \leqslant f_{2} \Rightarrow \mathcal{N}\left(f_{1}\right) \leqslant \mathcal{N}\left(f_{2}\right)
$$

and that for any sub- $\sigma$-field $\mathcal{T}$ of $\mathcal{S}$, the conditional expectation $\mathbb{E}_{\mathcal{T}}$ with respect to $\mathcal{T}$ is a bounded endomorphism of $\mathbb{L}^{\mathcal{N}}$ : there exists $K \in[1,+\infty)$ such that

$$
\forall f \in \mathbb{L}^{\mathcal{N}}, \quad \mathcal{N}\left(\mathbb{E}_{\mathcal{T}}[f]\right) \leqslant K \mathcal{N}(f)
$$

The first assumption enables us to replace (8) by

$$
\begin{aligned}
\mathcal{N}\left(M\left[\mathbb{1}_{A_{k}}\right]\right) & \geqslant\left(1-2 \delta_{n}\right) \mathcal{N}\left(\mathbb{1}_{B_{k}}\right) \\
& \geqslant\left(1-2 \delta_{n}\right) k(n)\left\|\mathbb{1}_{B_{k}}\right\|_{\mathbb{L}^{2}(\mu)} \\
& \geqslant \frac{1-2 \delta_{n}}{\sqrt{2}} k(n)\left\|\mathbb{1}_{A_{k}}\right\|_{\mathbb{L}^{2}(\mu)}
\end{aligned}
$$

where

$$
k(n):=\sup \left\{\frac{\mathcal{N}\left(\mathbb{1}_{B}\right)}{\sqrt{\mu(B)}}: 0<\mu(B) \leqslant \frac{1}{n}\right\}
$$

The second property plays the role of Jensen's inequality, so that instead of (9), we get for any $g \in \mathbb{L}^{2}\left(\mu_{N}\right)$,

$$
\mathcal{N}\left(I_{N} M_{N}[g]\right) \leqslant K \mathcal{N}\left(M I_{N}[g]\right)
$$

Since these are the only features of the $\mathbb{L}^{p}$ norms that we have used to prove Theorem 1 , we deduce the following extension:

Theorem 9 Under the hypothesis that $\lim _{n \rightarrow \infty} k(n)=+\infty$, meaning in some sense that $\mathcal{N}$ is stronger than the $\mathbb{L}^{2}$ norm, Theorem 1 is still valid if hyperboundedness is replaced by the fact that $\|M\|_{\mathbb{L}^{2}(\mu) \rightarrow \mathbb{L}^{\mathcal{N}}}<+\infty$. 
A typical instance of the above situation is given by Orlicz's norms (for a convenient summary, see e.g. Chapter 9 of Neveu [29]). Let $\Phi: \mathbb{R}_{+} \rightarrow \mathbb{R}_{+}$be a Young function: it is continuous, non-decreasing, convex and vanishes at 0 . The corresponding norm $\mathcal{N}$ is given by

$$
\forall f \in \mathcal{F}, \quad \mathcal{N}(f):=\inf \left\{a>0: \mu\left[\Phi\left(\frac{|f|}{a}\right)\right] \leqslant 1\right\}
$$

The previous requirements on $\mathcal{N}$ are met, since Jensen's is also satisfied in this setting, namely (22) is valid with $K=1$. The main hypothesis, $\lim _{n \rightarrow \infty} k(n)=+\infty$, of the above theorem then amounts to

$$
\lim _{r \rightarrow+\infty} \frac{\sqrt{r}}{\Phi^{-1}(r)}=+\infty
$$

or equivalently,

$$
\lim _{r \rightarrow+\infty} \frac{\Phi(r)}{r^{2}}=+\infty
$$

\section{Quantitative links between hyperboundedness and spectrum}

It will be checked that Theorem 1 does not admit a general quantitative version relative to the spectral gap, instead it is possible to give a bound on another eigenvalue. We will also see how the classical hypercontractivity of the Ornstein-Ulhenbeck process enables to recover that some estimates of Lee, Gharan and Trevisan [23] are optimal.

We begin by considering the $\mathbb{L}^{2}$ to $\mathbb{L}^{4}$ hypercontractivity. In [34], Wang has shown that if $\|M\|_{\mathbb{L}^{2}(\mu) \rightarrow \mathbb{L}^{4}(\mu)}^{4}<2$, then Theorem 1 is true and it is possible to deduce a lower bound on the spectral gap in term of $\|M\|_{\mathbb{L}^{2}(\mu) \rightarrow \mathbb{L}^{4}(\mu)}$. In next result we show that this fact cannot be extended in general.

Proposition 10 For any $K \geqslant 2$ and for any $\epsilon \in(0,1)$, we can find a self-adjoint ergodic Markov operator $M$ whose spectral gap is $\epsilon$ and which is such that $\|M\|_{\mathbb{L}^{2}(\mu) \rightarrow \mathbb{L}^{4}(\mu)}^{4}=K$.

As in Wang [34], we construct the example on the two points state space $S:=\{0,1\}$, but we rather consider all the probability measures $\mu:=(\eta, 1-\eta)$, with $\eta \in(0,1 / 2]$. For $\epsilon \in[0,1]$, we are interested in the self-adjoint Markov operator in $\mathbb{L}^{2}(\mu)$ given by

$$
M:=(1-\epsilon) \operatorname{Id}+\epsilon \mu
$$

(where $\mu$ is interpreted as the Markov operator associating $\mu[f] \mathbb{1}$ to any function $f \in \mathbb{L}^{2}(\mu)$ ). The spectrum of $M$ is constituted of the two eigenvalues 1 and $1-\epsilon$, so that if $\epsilon>0, M$ is ergodic and its spectral gap of $M$ is $\epsilon$. We define

$$
\forall(\eta, \epsilon) \in(0,1 / 2] \times[0,1], \quad F(\eta, \epsilon):=\|M\|_{\mathbb{L}^{2}(\mu) \rightarrow \mathbb{L}^{4}(\mu)}^{4}
$$

Lemma 11 The above mapping $F$ is continuous on $(0,1 / 2] \times[0,1]$. Furthermore it satisfies

$$
\begin{aligned}
& \max \{F(1 / 2, \epsilon): \epsilon \in[0,1]\}=2 \\
& \forall \epsilon \in(0,1), \quad \lim _{\eta \rightarrow 0_{+}} F(\eta, \epsilon)=+\infty
\end{aligned}
$$




\section{Proof}

Let $\varphi$ be the function defined on $S$ by $\varphi(0):=-\sqrt{(1-\eta) \eta}$ and $\varphi(1)=\sqrt{\eta /(1-\eta)}$, so that $(\mathbb{1}, \varphi)$ is an orthonormal basis of $\mathbb{L}^{2}(\mu)$ diagonalizing $M$. Consider $f:=x \mathbb{1}+y \varphi$ with $x, y \in \mathbb{R}$. Of course we have $\mu\left[f^{2}\right]=x^{2}+y^{2}$ and we compute that

$$
\begin{aligned}
\mu\left[(M[f])^{4}\right] & =\mu\left[(x \mathbb{1}+(1-\epsilon) y \varphi)^{4}\right] \\
& =x^{4}+A(\eta, \epsilon) x^{2} y^{2}+B(\eta, \epsilon) x y^{3}+C(\eta, \epsilon) y^{4}
\end{aligned}
$$

where for any $(\eta, \epsilon) \in(0,1 / 2] \times[0,1]$,

$$
\begin{aligned}
A(\eta, \epsilon) & :=6(1-\epsilon)^{2} \\
B(\eta, \epsilon) & :=4(1-\epsilon)^{3} \frac{2 \eta-1}{\sqrt{\eta(1-\eta)}} \\
C(\eta, \epsilon) & :=(1-\epsilon)^{4} \frac{1-3 \eta+3 \eta^{2}}{\eta(1-\eta)}
\end{aligned}
$$

Thus we have

$$
F(\eta, \epsilon)=\max \left\{x^{4}+A(\eta, \epsilon) x^{2} y^{2}+B(\eta, \epsilon) x y^{3}+C(\eta, \epsilon) y^{4}: x^{2}+y^{2}=1\right\}
$$

The continuity of $F$ is a direct consequence of the continuity of $A, B$ and $C$ and of the compactness of the circle $\left\{(x, y) \in \mathbb{R}^{2}: x^{2}+y^{2}=1\right\}$. By considering its point $(x, y)=(0,1)$, it appears that $F \geqslant C$, from which follows the last assertion of the lemma. Finally, we have

$$
\forall \epsilon \in[0,1], \quad F(1 / 2, \epsilon)=\max \left\{x^{4}+6(1-\epsilon)^{2} x^{2} y^{2}+(1-\epsilon)^{4} y^{4}: x^{2}+y^{2}=1\right\}
$$

The r.h.s. is decreasing as a function of $\epsilon \in[0,1]$, so that

$$
\begin{aligned}
\max \{F(1 / 2, \epsilon): \epsilon \in[0,1]\} & =F(1 / 2,0) \\
& =\max \left\{x^{2}+6 x(1-x)+(1-x)^{2}: x \in[0,1]\right\} \\
& =2
\end{aligned}
$$

Proposition 10 is an immediate consequence of the above lemma: for any $K \geqslant 2$ and $\epsilon \in(0,1)$, consider the mapping $(0,1 / 2] \ni \eta \mapsto F(\eta, \epsilon)$ to find $\eta \in(0,1 / 2]$ such that $F(\eta, \epsilon)=K$. The corresponding Markov operator $M$ satisfies the requirements of Proposition 10.

Returning to the general case, the proofs of the previous sections only provide a bound on a certain eigenvalue, not necessarily the spectral gap. Since we are now looking for a quantitative bound, we must use the sharpest result obtained by Lee, Gharan and Trevisan [23], namely their Theorem 4.1, one consequence of which can be written as:

Theorem 12 ([23]) With the notations of Section 2, there exists a universal constant $\eta>0$ such that if $c(n):=\eta / \ln (1+n)$, then for any finite self-adjoint Markov operator,

$$
\forall n \in \mathbb{N}, \quad \lambda_{2 n} \geqslant c(n) \iota_{n}^{2}
$$

As it was explained in Sections 3 and 4, the finiteness assumption can be removed by approximation. Furthermore the above result admits an immediate extension to the compact Riemannian framework as in Theorem 7 .

We can now come back to the setting of Section 5 and consider a Young function $\Phi$ and the corresponding quantities defined in (23) and whose values are given by

$$
\forall n \in \mathbb{N}, \quad k(n)=\frac{\sqrt{n}}{\Phi^{-1}(n)}
$$


(at least if $\mu$ has no atom, otherwise only an inequality holds, but for our purposes we can take the $k(n), n \in \mathbb{N}$, to be the corresponding upper bounds). Revisiting the arguments of Section 2 (with $\delta_{n}=1 / 4$ for $n \in \mathbb{N}$ ) and taking into account the observations of Sections 4 and 5 and Theorem 12, we get the following quantitative version of Theorem 1:

Theorem 13 Let $M$ be a self-adjoint Markov operator. If $n \in \mathbb{N}$ is such that $k(n) \geqslant 2 \sqrt{2}\|M\|_{\mathbb{L}^{2} \rightarrow \mathbb{L}^{\Phi}}$, then we are assured of

$$
\lambda_{2 n}(M) \geqslant \frac{c(n)}{16}
$$

where for any $m \in \mathbb{N}$, the quantities $k(m), c(m)$ and $\lambda_{m}(M)$ are defined in (24), Theorem 12 and (11). Thus the top of the spectrum of $M$ consists of $2 n$ eigenvalues $1,1-\lambda_{2}(M), \ldots, 1-\lambda_{2 n}(M)$ (with multiplicities: some of them can be equal). In particular, Theorem 1 is recovered if $M$ is furthermore assumed to be ergodic.

It is tempting to investigate what happens to this bound when we rather start with a selfadjoint Markov semi-group $\left(P_{t}\right)_{t \geqslant 0}$. Let us consider the most famous example of hypercontractive semi-group, the Ornstein-Uhlenbeck process, first on $\mathbb{R}$. Later on, we will tensorize it to verify that in general the order of the estimate of Theorem 13 cannot be improved and by consequence this is also true for Theorem 12. In Section 4.3 of [23], Lee, Gharan and Trevisan applied other hypercontractivity results due to Bonami [6] and Beckner [5] to noisy hypercubes in order to check that for large $n \in \mathbb{N}$ the order of $c(n)$ given in Theorem 12 is optimal.

So let $\left(P_{t}\right)_{t \geqslant 0}$ be the self-adjoint Markov semi-group associated to the Ornstein-Uhlenbeck generator defined by

$$
\forall f \in \mathcal{C}_{b}^{2}(\mathbb{R}), \forall x \in \mathbb{R}, \quad L[f](x):=f^{\prime \prime}(x)-x f^{\prime}(x)
$$

which is essentially self-adjoint on $\mathbb{L}^{2}(\gamma)$, where $\gamma$ is the normal centered Gaussian distribution. It is well-known (see Nelson [28] and Gross [14]) that for any $p>2$,

$$
\left\|P_{t}\right\|_{\mathbb{L}^{2}(\gamma) \rightarrow \mathbb{L}^{p}(\gamma)}= \begin{cases}+\infty & , \text { if } t<\frac{1}{2} \ln (p-1) \\ 1 & , \text { if } t \geqslant \frac{1}{2} \ln (p-1)\end{cases}
$$

Applying Theorem 13 with $M=P_{t}$ for $t>0$ and relatively to the usual Lebesgue space $\mathbb{L}^{p}(\gamma)$ with $p>2$, we get that for any $n \in \mathbb{N}$,

$$
\left\{\begin{array}{rl}
n^{\frac{1}{2}-\frac{1}{p}} & \geqslant 2 \sqrt{2} \\
t & \geqslant \frac{1}{2} \ln (p-1)
\end{array} \quad \Rightarrow 1-\exp \left(-t \lambda_{2 n}(L)\right) \geqslant \frac{c(n)}{16}\right.
$$

where $c(n)$ is defined in Theorem 12. This leads us to define for $n \in \mathbb{N}$,

$$
\begin{aligned}
p_{n} & :=\frac{2 \ln (n)}{\ln (n)-2 \ln (2 \sqrt{2})} \\
t_{n} & :=\frac{1}{2} \ln \left(p_{n}-1\right) \\
& =\frac{1}{2} \ln \left(\frac{\ln (n)+2 \ln (2 \sqrt{2})}{\ln (n)-2 \ln (2 \sqrt{2})}\right)
\end{aligned}
$$

and to consider $n_{0} \in \mathbb{N}$ the smallest integer such that $p_{n}>2$. Taking into account the convexity bound $s \geqslant 1-\exp (-s)$ for any $s \in \mathbb{R}$, we deduce that

$$
\forall n \in \mathbb{N}, \quad n \geqslant n_{0} \Longrightarrow \lambda_{n}(L) \geqslant \frac{c(n)}{16 t_{n}}
$$


Since for large $n \in \mathbb{N}, t_{n} \sim 2 \ln (2 \sqrt{2}) / \ln (n) \sim 2 \ln (2 \sqrt{2}) \eta c(n)$, this result essentially means that $\lambda_{n}(L)$ is bounded below for $n \geqslant n_{0}$. It seems quite disappointing, since it is well-known that $\lambda_{n}(L)=n-1$ for all $n \in \mathbb{N}$. So we could try to improve Theorem 12 by obtaining quantities $c(n)$, $n \in \mathbb{N}$, satisfying $\lim _{n \rightarrow \infty} c(n) \ln (n)=+\infty$, since it would lead to a lower bound in the r.h.s. of (26) going to infinity as $n \rightarrow \infty$.

But this is not possible, because the previous arguments are stable by tensorization. More precisely, for $N \in \mathbb{N} \sqcup\{\infty\}$, consider the semi-group $\left(P_{t}^{\otimes N}\right)_{t \geqslant 0}$ acting on $\mathbb{L}^{2}\left(\gamma^{\otimes N}\right)$. The same hypercontractivity property (25) is valid for this semi-group. The generator $L^{(N)}$ of $\left(P_{t}^{\otimes N}\right)_{t \geqslant 0}$ corresponds to the sum of $N$ copies of $L$, each acting on different coordinates of $\mathbb{R}^{N}$. In particular, we get

$$
\forall n \in \llbracket 2, N+1 \rrbracket, \quad \lambda_{n}\left(L^{(N)}\right)=\lambda_{2}(L)
$$

This forbids the r.h.s. of (26) to be improved into a lower bound going to infinity with $n$. In particular the lower bound of Lee, Gharan and Trevisan [23] presented in Theorem 12 is of optimal order.

\section{A On principal Dirichlet eigenvalues at small temper- ature}

The goal of this appendix is to check the assertions (17), (18), (19) and (20) presented in Section 4. Concerning the three first relations, we will adapt the proofs of Holley, Kusuoka and Stroock [21] for the corresponding results relative to the spectral gap of a Witten Laplacian on a compact Riemannian manifold without boundary. Their computations are based on the following ideas. The upper bound is obtained by considering a function approximating the indicator function of a well whose height is maximum among those not intersecting a fixed global minimum of the potential. For the Dirichlet eigenvalues, the argument is even simpler, by using a well of maximum height included in $A$ or a small ball if there is no such well. Concerning the lower bound, Holley, Kusuoka and Stroock [21] consider paths with minimal elevation connecting generical points of the manifold with a fixed global minimum of the potential. This approach can in principle be applied to the Dirichlet eigenvalues by using paths with minimal elevation connecting generical points of $A$ to $\partial A$. But technically it requires some curvature bounds on $\partial A$, so we preferred to resort to a modified elevation and to paths linking generical points of $A$ to some nice interior points of $A^{\mathrm{c}}$. The advantage is that the existence of such points is not really restrictive when one is computing Dirichlet connectivity spectra, as shown by (20).

The notations are those introduced in Section 4. We begin by considering the simplest bounds:

\section{Proof of (18) and (19)}

It is based, on one hand on the observation that for any fixed $\beta \geqslant 0$, the mapping $\widehat{\mathcal{D}}_{1} \ni A \mapsto$ $\lambda_{0}\left(A, L_{\beta}\right)$ is non-increasing, when $\widehat{\mathcal{D}}_{1}$ is endowed with the inclusion order. And on the other hand on the fact that for any $\beta \geqslant 0$ and $B \in \widehat{\mathcal{D}}_{1}$,

$$
\exp \left(-\beta\left(\sup _{B} U-\inf _{B} U\right)\right) \lambda_{0}\left(L_{0}, B\right) \leqslant \lambda_{0}\left(L_{\beta}, B\right) \leqslant \exp \left(\beta\left(\sup _{B} U-\inf _{B} U\right)\right) \lambda_{0}\left(L_{0}, B\right)
$$

If $B$ is a cycle, we have $h(B)=\sup _{B} U-\inf _{B} U$, so (18) follows at once. If $B$ is a ball of radius $r>0, \sup _{B} U-\inf _{B} U \leqslant 2 r\|\nabla U\|_{\infty} \leqslant 2 r$, so that we get for any $A \in \widehat{\mathcal{D}}_{1}$,

$$
\lambda_{0}\left(L_{\beta}, A\right) \leqslant e^{2} \max \left\{\lambda_{0}\left(L_{0}, B\right): B \subset A \text { is a ball of radius less than } 1 / \beta\right\}
$$

To conclude to (19), it remains to remark that by compactness of $S$, there exists a constant $k_{S}^{(3)} \geqslant 1$, depending only on the Riemannian structure of $S$, such that

$$
\forall x \in S, \forall r \in(0,1], \quad\left(k_{S}^{(3)}\right)^{-1} r^{-2} \leqslant \lambda_{0}\left(L_{0}, B(x, r)\right) \leqslant k_{S}^{(3)} r^{-2}
$$


The following arguments mainly follow those of Holley, Kusuoka and Stroock [21]. We will adopt their notations (sometimes with a $A$ in index when the corresponding notions differ), so that we can refer directly to their proof.

Proof of (17)

Let $\beta \geqslant 1$ be fixed as well as a set $A \in \widehat{\mathcal{D}}_{1}(1 / \beta)$. We modify the potential $U$ by defining

$$
\forall x \in S, \quad U_{A}(x):= \begin{cases}U(x) & , \text { if } x \in A \sqcup \partial A \\ -\infty & \text { otherwise }\end{cases}
$$

The elevation $E_{A}(\gamma)$ of a path $\gamma \in \mathcal{C}([0,1], S)$ is $E_{A}(\gamma)=\max _{t \in[0,1]} U_{A}(\gamma(t))$ and for $x, y \in S$ define

$$
H_{A}(x, y):=\inf \left\{E_{A}(\gamma): \gamma \in \mathcal{C}([0,1], S), \gamma(0)=x, \gamma(1)=y\right\}
$$

There is no difficulty in checking that

$$
\begin{aligned}
h(A) & =\max \left\{H_{A}(x, y)-U(x): x \in A, y \notin A\right\} \\
& =\max _{x \in A} \min _{j \in \llbracket J \rrbracket} H_{A}\left(x, y_{j}\right)-U(x)
\end{aligned}
$$

where $J$ is the number of connected components of $A^{\text {c }}$ (there are only a finite number of them by the restrictions imposed on $\left.\widehat{\mathcal{D}}_{1}\right)$ and where the $y_{j}, j \in \llbracket J \rrbracket$, are any choice of points in each of them, but we take them satisfying

$$
\mu\left(B\left(y_{j}, 1 / \beta\right) \cap A^{\mathrm{c}}\right) \geqslant \frac{\mu\left(B\left(y_{j}, 1 / \beta\right)\right)}{2}
$$

For any $\beta \geqslant 1$, there exists a finite cover of $A$ by balls $\left\{B\left(x_{k}, 1 / \beta\right): k \in \llbracket N_{\beta} \rrbracket\right\}$, where the $x_{k}$, $k \in \llbracket N_{\beta} \rrbracket$, are points of $A$, where $N_{\beta} \leqslant k_{S}^{(4)} \beta^{\operatorname{dim}(S)}$, with $k_{S}^{(4)}<+\infty$ is a constant depending only on $S$ and not on $A$. Denote $Z_{\beta}:=\int \exp (-\beta U) d \mu$.

Considering any function $\phi \in \mathcal{C}^{1}(S)$ vanishing on $A^{\mathrm{c}}$, we can write

$$
\begin{aligned}
\mu_{\beta}\left[\phi^{2}\right] & \leqslant \frac{1}{Z_{\beta}} \sum_{k \in \llbracket N_{\beta} \rrbracket} \int_{B\left(x_{k}, 1 / \beta\right)} \exp (-\beta U(x)) \phi^{2}(x) \mu(d x) \\
& =\frac{1}{Z_{\beta} \mu\left(B\left(y_{j(k)}, 1 / \beta\right) \cap A^{\mathrm{c}}\right)} \sum_{k \in \llbracket N_{\beta} \rrbracket} \int_{B\left(x_{k}, 1 / \beta\right) \times\left(B\left(y_{j(k)}, 1 / \beta\right) \cap A^{\mathrm{c}}\right)}^{\exp (-\beta U(x))(\phi(x)-\phi(y))^{2} \mu(d x) \mu(d y)} \\
& \leqslant \frac{2}{Z_{\beta} \mu\left(B\left(y_{j(k)}, 1 / \beta\right)\right)} \sum_{k \in \llbracket N_{\beta} \rrbracket} \int_{B\left(x_{k}, 1 / \beta\right) \times B\left(y_{j(k)}, 1 / \beta\right)}^{\exp (-\beta U(x))(\phi(x)-\phi(y))^{2} \mu(d x) \mu(d y)} \\
& \leqslant \frac{2 e k_{S}^{(5)} \beta^{\operatorname{dim}(S)}}{Z_{\beta}} \sum_{k \in \llbracket N_{\beta} \rrbracket} \exp \left(-\beta U\left(x_{k}\right)\right) \int_{B\left(x_{k}, 1 / \beta\right) \times B\left(y_{j(k)}, 1 / \beta\right)}^{(\phi(x)-\phi(y))^{2}} \mu(d x) \mu(d y)
\end{aligned}
$$

where $j(k) \in \llbracket J \rrbracket$ is such that

$$
H_{A}\left(x_{k}, y_{j(k)}\right)=\min _{j \in \llbracket \rrbracket} H_{A}\left(x_{k}, y_{j}\right)
$$

and where $k_{S}^{(5)} \geqslant 1$ is a constant only depending on the structure of $S$ such that

$$
\forall x \in S, \forall r \in(0,1], \quad\left(k_{S}^{(5)}\right)^{-1} r^{\operatorname{dim}(S)} \leqslant \mu(B(x, r)) \leqslant k_{S}^{(5)} r^{\operatorname{dim}(S)}
$$


With these preliminaries, the proof is now identical to that of Holley, Kusuoka and Stroock developed in pages 338 to 340 of [21]. They find a constant $k_{S}^{(6)}>0$, only depending on $S$, such that for any $\beta \geqslant 1$ and $\phi$ as above,

$$
\begin{gathered}
\sup \left\{\exp (-\beta U(x)) \int_{B(x, 1 / \beta) \times B(y, 1 / \beta)}(\phi(w)-\phi(v))^{2} \mu(d v) \mu(d w): x \in A, y \notin A\right\} \\
\leqslant k_{S}^{(6)} \beta^{2(\operatorname{dim}(S)-1)} Z_{\beta} \exp (\beta h(A)) \int\langle\nabla \phi, \nabla \phi\rangle d \mu_{\beta}
\end{gathered}
$$

Putting together the above computations, we end up with the Poincaré inequality

$$
\mu_{\beta}\left[\phi^{2}\right] \leqslant 2 e k_{S}^{(4)} k_{S}^{(5)} k_{S}^{(6)} \beta^{4 \operatorname{dim}(S)-2} \exp (\beta h(A)) \int\langle\nabla \phi, \nabla \phi\rangle d \mu_{\beta}
$$

whose validity for all $\mathcal{C}^{1}$ functions $\phi$ vanishing outside $A$ implies (17).

The proof of the remaining bound will justify the restrictions imposed on $\widehat{\mathcal{D}}_{n}$, which could have looked strange at first view.

\section{Proof of (20)}

So let be given $n \geqslant 2, \beta \geqslant 1$ and $\left(A_{1}, \ldots, A_{n}\right) \in \widehat{\mathcal{D}}_{n}$ such that $A_{1}$ does not belong to $\widehat{\mathcal{D}}_{1}(1 / \beta)$, we are going to prove that there exists $k \in \llbracket 2, n \rrbracket$ with

$$
\lambda_{0}\left(L_{\beta}, A_{k}\right) \geqslant k_{S}^{\prime \prime} \beta^{2}
$$

where $k_{S}^{\prime \prime}>0$ is a constant depending only on the Riemannian structure of $S$. Indeed, by definition of $\widehat{\mathcal{D}}_{1}(1 / \beta)$, there exists a connected component of $A^{\mathrm{c}}$ which is such that all its points $x$ satisfy

$$
\mu\left(B(x, 1 / \beta) \cap A_{1}\right)>\frac{\mu(B(x, 1 / \beta))}{2}
$$

By definition of $\widehat{\mathcal{D}}_{n}$, this connected component contains a subset $A_{k}$, with $k \in \llbracket 2, n \rrbracket$, it is the one that will satisfy $(27)$. Consider $\left(X_{t}^{(\beta)}\right)_{t \geqslant 0}$ a diffusion process of generator $L_{\beta}$ and denote $\tau:=\inf \left\{t \geqslant 0: X_{t}^{(\beta)} \notin A_{k}\right\}$. Since $L_{\beta}$ is elliptic and $A_{k}$ is a connected open set, $\lambda_{0}\left(L_{\beta}, A_{k}\right)$ is the asymptotic rate of getting out of $A_{k}$ :

$$
\forall x \in A_{k}, \quad \lambda_{0}\left(L_{\beta}, A_{k}\right)=-\lim _{t \rightarrow+\infty} \frac{1}{t} \ln \left(\mathbb{P}_{x}[\tau>t]\right)
$$

where the $x$ in $\mathbb{P}_{x}$ indicates that the diffusion is starting from $x: \mathbb{P}_{x}$-almost surely, $X_{0}^{(\beta)}=x$. Thus taking into account the Markov property, to get (27), it is sufficient to find another constant $k_{S}^{(7)}>0$ depending only on $S$ such that

$$
\forall x \in A_{k}, \quad \mathbb{P}_{x}\left[\tau \leqslant 1 / \beta^{2}\right] \geqslant k_{S}^{(7)}
$$

It is even enough to show that

$$
\forall x \in A_{k}, \quad \mathbb{P}_{x}\left[X_{1 / \beta^{2}}^{(\beta)} \in A_{1}\right] \geqslant k_{S}^{(7)}
$$

Denote by $\left(p_{t}^{(\beta)}(x, y)\right)_{t>0, x, y \in S}$ the kernels corresponding to the semi-group associated to the generator $L_{\beta}$, so we can write

$$
\mathbb{P}_{x}\left[X_{1 / \beta^{2}}^{(\beta)} \in A_{1}\right]=\int_{A_{1}} p_{1 / \beta^{2}}^{(\beta)}(x, y) \mu(d y)
$$


From Theorem 3.1 of Wang [33], we have that for any $t>0, \sigma>0$ and $x, y \in S$,

$$
p_{t}^{(\beta)}(x, y) \geqslant k_{S}^{(8)}(2 \pi t)^{-\operatorname{dim}(S) / 2} \exp \left[-\left(\frac{1}{2 t}+\frac{\sigma}{3 \sqrt{t}}\right) \rho^{2}(x, y)-\frac{b^{2} t}{8}-\left(\frac{b^{2}}{4 \sigma}+\frac{2 \operatorname{dim}(S) \sigma}{3}\right) \sqrt{t}\right]
$$

where $k_{S}^{(8)}>0$ is the volume of $S$ (with respect to the unnormalized Riemannian measure of $S$ ), where $\rho(x, y)$ is the Riemannian distance between $x$ and $y$ and where $b:=K_{S}+\beta, K_{S} \geqslant 0$ being such that the Ricci curvature of $S$ is bounded below by $-K_{S}$. In particular if we choose $\sigma=\beta$, it appears there exists a constant $k_{S}^{(9)}>0$, only depending on $S$, such that

$$
\forall x \in S, \forall y \in B(x, 1 / \beta), \quad p_{1 / \beta^{2}}^{(\beta)}(x, y) \geqslant k_{S}^{(9)} \beta^{\operatorname{dim}(S)}
$$

It follows that for all $x \in A_{k}$,

$$
\begin{aligned}
\int_{A_{1}} p_{1 / \beta^{2}}^{(\beta)}(x, y) \mu(d y) & \geqslant \int_{A_{1} \cap B(x, 1 \beta)} p_{1 / \beta^{2}}^{(\beta)}(x, y) \mu(d y) \\
& \geqslant k_{S}^{(9)} \beta^{\operatorname{dim}(S)} \mu\left(A_{1} \cap B(x, 1 \beta)\right) \\
& \geqslant \frac{k_{S}^{(9)}}{2} \beta^{\operatorname{dim}(S)} \mu(B(x, 1 \beta)) \\
& \geqslant \frac{k_{S}^{(9)}}{2 k_{S}^{(5)}}
\end{aligned}
$$

as required.

\section{Aknowledgments:}

I would like to express my gratefulness toward Feng-Yu Wang, who presented to me the conjecture of Høegh-Krohn and Simon and explained me his works on the subject, as well as to Bernard Helffer, who pointed out some shortages around (17) in a first version of this manuscript.

\section{References}

[1] Shigeki Aida. Uniform positivity improving property, Sobolev inequalities, and spectral gaps. J. Funct. Anal., 158(1):152-185, 1998.

[2] N. Alon. Eigenvalues and expanders. Combinatorica, 6(2):83-96, 1986. Theory of computing (Singer Island, Fla., 1984).

[3] N. Alon and V. D. Milman. $\lambda_{1}$, isoperimetric inequalities for graphs, and superconcentrators. J. Combin. Theory Ser. B, 38(1):73-88, 1985.

[4] Cécile Ané, Sébastien Blachère, Djalil Chafaï, Pierre Fougères, Ivan Gentil, Florent Malrieu, Cyril Roberto, and Grégory Scheffer. Sur les inégalités de Sobolev logarithmiques, volume 10 of Panoramas et Synthèses [Panoramas and Syntheses]. Société Mathématique de France, Paris, 2000. With a preface by Dominique Bakry and Michel Ledoux.

[5] William Beckner. Inequalities in Fourier analysis. Ann. of Math. (2), 102(1):159-182, 1975.

[6] Aline Bonami. Étude des coefficients de Fourier des fonctions de $L^{p}(G)$. Ann. Inst. Fourier (Grenoble), 20(fasc. 2):335-402 (1971), 1970.

[7] Anton Bovier, Véronique Gayrard, and Markus Klein. Metastability in reversible diffusion processes. II. Precise asymptotics for small eigenvalues. J. Eur. Math. Soc. (JEMS), 7(1):6999, 2005. 
[8] Patrick Cattiaux. A pathwise approach of some classical inequalities. Potential Anal., 20(4):361-394, 2004.

[9] Jeff Cheeger. A lower bound for the smallest eigenvalue of the Laplacian. In Problems in analysis (Papers dedicated to Salomon Bochner, 1969), pages 195-199. Princeton Univ. Press, Princeton, N. J., 1970.

[10] Amir Daneshgar, Ramin Javadi, and Laurent Miclo. On nodal domains and higherorder Cheeger inequalities of finite reversible Markov processes. Stochastic Process. Appl., 122(4):1748-1776, 2012.

[11] M. I. Freidlin and A. D. Wentzell. Random perturbations of dynamical systems, volume 260 of Grundlehren der Mathematischen Wissenschaften [Fundamental Principles of Mathematical Sciences]. Springer-Verlag, New York, 1984. Translated from the Russian by Joseph Szücs.

[12] Masatoshi Fukushima, Yōichi Ōshima, and Masayoshi Takeda. Dirichlet forms and symmetric Markov processes, volume 19 of de Gruyter Studies in Mathematics. Walter de Gruyter \& Co., Berlin, 1994.

[13] Fuzhou Gong and Liming Wu. Spectral gap of positive operators and applications. J. Math. Pures Appl. (9), 85(2):151-191, 2006.

[14] Leonard Gross. Logarithmic Sobolev inequalities. Amer. J. Math., 97(4):1061-1083, 1975.

[15] B. Helffer, T. Hoffmann-Ostenhof, and S. Terracini. Nodal domains and spectral minimal partitions. Ann. Inst. H. Poincaré Anal. Non Linéaire, 26(1):101-138, 2009.

[16] Bernard Helffer. Semiclassical analysis, Witten Laplacians, and statistical mechanics, volume 1 of Series in Partial Differential Equations and Applications. World Scientific Publishing Co. Inc., River Edge, NJ, 2002.

[17] Bernard Helffer, Markus Klein, and Francis Nier. Quantitative analysis of metastability in reversible diffusion processes via a Witten complex approach. Mat. Contemp., 26:41-85, 2004.

[18] Bernard Helffer and Francis Nier. Quantitative analysis of metastability in reversible diffusion processes via a Witten complex approach: the case with boundary. Mém. Soc. Math. Fr. (N.S.), (105):vi+89, 2006.

[19] Masanori Hino. Exponential decay of positivity preserving semigroups on $L^{p}$. Osaka J. Math., 37(3):603-624, 2000.

[20] Masanori Hino. Correction to: "Exponential decay of positivity preserving semigroups on $L^{p}$ " [Osaka J. Math. 37 (2000), no. 3, 603-624; MR1789439 (2001k:60109)]. Osaka J. Math., $39(3): 771,2002$.

[21] Richard A. Holley, Shigeo Kusuoka, and Daniel W. Stroock. Asymptotics of the spectral gap with applications to the theory of simulated annealing. J. Funct. Anal., 83(2):333-347, 1989.

[22] Gregory F. Lawler and Alan D. Sokal. Bounds on the $L^{2}$ spectrum for Markov chains and Markov processes: a generalization of Cheeger's inequality. Trans. Amer. Math. Soc., 309(2):557-580, 1988.

[23] J. R. Lee, S. Oveis Gharan, and L. Trevisan. Multi-way spectral partitioning and higher-order Cheeger inequalities. ArXiv e-prints, November 2011.

[24] Anand Louis, Prasad Raghavendra, Prasad Tetali, and Santosh Vempala. Finding sparse cuts via Cheeger inequalities for higher eigenvalues. Preprint available on http://people.math.gatech.edu/ tetali/pubs_2012.html under the name Many Sparse Cuts via Higher Eigenvalues.

[25] P. Mathieu. Quand l'inégalité log-Sobolev implique l'inégalité de trou spectral. In Séminaire de Probabilités, XXXII, volume 1686 of Lecture Notes in Math., pages 30-35. Springer, Berlin, 1998. 
[26] Laurent Miclo. Comportement de spectres d'opérateurs de Schrödinger à basse température. Bull. Sci. Math., 119(6):529-553, 1995.

[27] Laurent Miclo. On eigenfunctions of Markov processes on trees. Probab. Theory Related Fields, 142(3-4):561-594, 2008.

[28] Edward Nelson. The free Markoff field. J. Functional Analysis, 12:211-227, 1973.

[29] Jacques Neveu. Martingales à temps discret. Masson et Cie, éditeurs, Paris, 1972.

[30] Michael Reed and Barry Simon. Methods of modern mathematical physics. I. Academic Press Inc. [Harcourt Brace Jovanovich Publishers], New York, second edition, 1980. Functional analysis.

[31] Barry Simon and Raphael Høegh-Krohn. Hypercontractive semigroups and two dimensional self-coupled Bose fields. J. Functional Analysis, 9:121-180, 1972.

[32] Alistair Sinclair and Mark Jerrum. Approximate counting, uniform generation and rapidly mixing Markov chains. Inform. and Comput., 82(1):93-133, 1989.

[33] Feng-Yu Wang. Sharp explicit lower bounds of heat kernels. Ann. Probab., 25(4):1995-2006, 1997.

[34] Feng-Yu Wang. Spectral gap for hyperbounded operators. Proc. Amer. Math. Soc., 132(9):2629-2638 (electronic), 2004.

[35] Feng-Yu Wang. Functional Inequalities, Markov Semigroups and Spectral Theory. Science Press, Beijing, New York, 2005.

[36] Liming Wu. Uniformly integrable operators and large deviations for Markov processes. $J$. Funct. Anal., 172(2):301-376, 2000.

[37] Kōsaku Yosida. Functional analysis. Classics in Mathematics. Springer-Verlag, Berlin, 1995. Reprint of the sixth (1980) edition.

miclo@math.univ-toulouse.fr

Institut de Mathématiques de Toulouse

Université Paul Sabatier

118, route de Narbonne

31062 Toulouse Cedex 9, France 